
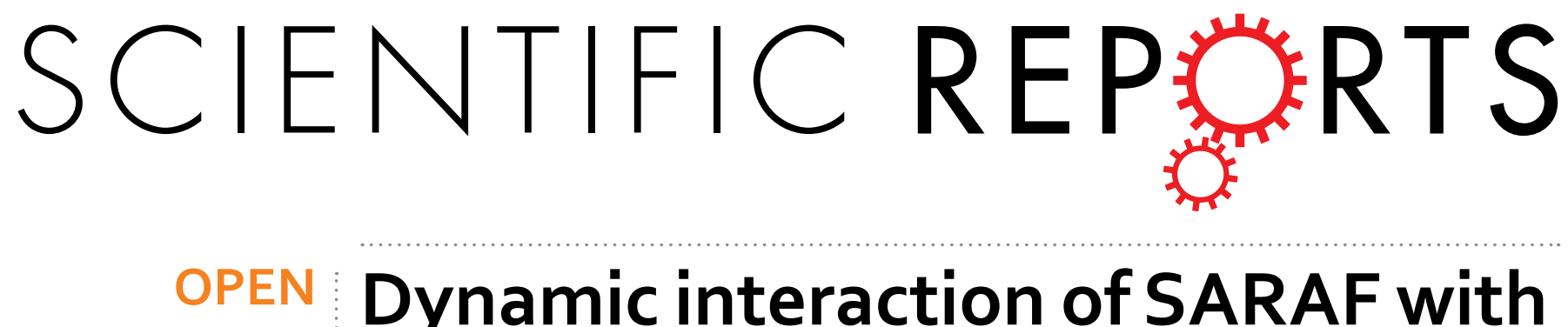

\title{
Dynamic interaction of SARAF with STIM1 and Orai1 to modulate store- operated calcium entry
}

Received: 27 September 2015

Accepted: 30 March 2016

Published: 12 April 2016

\section{Letizia Albarran ${ }^{1}$, Jose J. Lopez ${ }^{1}$, Nidhal Ben Amor ${ }^{1}$, Francisco E. Martin-Cano ${ }^{1}$, Alejandro Berna-Erro ${ }^{1}$, Tarik Smani ${ }^{2}$, Gines M. Salido ${ }^{1}$ \& Juan A. Rosado ${ }^{1}$}

$\mathrm{Ca}^{2+}$ influx by store-operated $\mathrm{Ca}^{2+}$ channels is a major mechanism for intracellular $\mathrm{Ca}^{2+}$ homeostasis and cellular function. Here we present evidence for the dynamic interaction between the SOCEassociated regulatory factor (SARAF), STIM1 and Orai1. SARAF overexpression attenuated SOCE and the STIM1-Orai1 interaction in cells endogenously expressing STIM1 and Orai1 while RNAi-mediated SARAF silencing induced opposite effects. SARAF impaired the association between Orai1 and the Orai1-activating small fragment of STIM1 co-expressed in the STIM1-deficient NG115-401L cells. Cell treatment with thapsigargin or physiological agonists results in direct association of SARAF with Orai1. STIM1-independent interaction of SARAF with Orai1 leads to activation of this channel. In cells endogenously expressing STIM1 and Orai1, $\mathrm{Ca}^{2+}$ store depletion leads to dissociation of SARAF with STIM1 approximately 30 s after treatment with thapsigargin, which paralleled the increase in SARAFOrai1 interaction, followed by reinteraction with STIM1 and dissociation from Orai1. Co-expression of SARAF and either Orai1 or various $\mathrm{N}$-terminal deletion Orai1 mutants did not alter SARAF-Orai1 interaction; however, expression of C-terminal deletion Orai1 mutants or blockade of the C-terminus of Orai1 impair the interaction with SARAF. These observations suggest that SARAF exerts an initial positive role in the activation of SOCE followed by the facilitation of SCDI of Orai1.

Store operated $\mathrm{Ca}^{2+}$ entry (SOCE) is a major pathway of calcium influx in non-excitable cells, and is essential for the activation of many cellular processes. This mechanism is initiated by the depletion of the intracellular $\mathrm{Ca}^{2+}$ stores, mainly the endoplasmic reticulum (ER). The two major components of SOCE are STIM1 $1^{1,2}$, the $\mathrm{Ca}^{2+}$ sensor of the ER that communicates the signal of store depletion to the plasma membrane resident $\mathrm{Ca}^{2+}$ channel, and the channel pore-forming Orai $1^{3-5}$.

Orail is a highly $\mathrm{Ca}^{2+}$-selective, inward-rectifying, channel that has been reported to be regulated by $\mathrm{Ca}^{2+}$ in two possible ways named fast $\mathrm{Ca}^{2+}$-dependent inactivation (FCDI), which occurs within milliseconds ${ }^{6,7}$, and slow $\mathrm{Ca}^{2+}$-dependent inactivation (SCDI) that inactivates Orail over 2-3 min to prevent $\mathrm{Ca}^{2+}$ overload ${ }^{8,9}$. The later mechanism is activated by $\mathrm{Ca}^{2+}$ entering through Orail channels, and constitutes a slow negative feedback process. SCDI has been reported to include both store refilling-dependent and independent components in Jurkat T cells ${ }^{8}$; although it has been found to be entirely independent on store refilling in rat basophilic leukemia cells ${ }^{9}$.

STIM1 N-terminus exhibits an EF-hand motif, which, upon $\mathrm{Ca}^{2+}$ dissociation, leads to oligomerization and clustering of STIM1 into puncta located at the ER-plasma membrane junctions ${ }^{10}$. This transition is accompanied by a conformational reorganization of its cytosolic region from a closed to an extended state leading to the exposition of the SOAR domain (amino acids $344-442^{11}$; also known as OASF $(233-450 / 474)^{12}$, CAD (342-448) $)^{13}$ or Ccb9 $\left.(339-444)^{14}\right)$, which results in full activation of Orai $1^{15}$. The crystal structure of SOAR has revealed that this domain exists as a V-shaped dimer ${ }^{16}$. In the resting state, STIM1 may exist mostly as dimers with the SOAR dimer likely occluded. In the inactive state, the $\mathrm{Ca}^{2+}$ bound intraluminal region remains monomeric ${ }^{10}$ but, upon $\mathrm{Ca}^{2+}$ store depletion, the $\mathrm{N}$-terminal region of STIM1 releases $\mathrm{Ca}^{2+}$ and oligomerizes resulting in the conformational change that releases the SOAR domain ${ }^{16}$.

Recent studies have identified a C-terminal inhibitory domain (CTID; amino acids 448-530) downstream of the SOAR domain, whose deletion leads to spontaneous clustering of STIM1 and activation of Orail in the absence of $\mathrm{Ca}^{2+}$ store depletion ${ }^{17}$. CTID has been reported to mediate the interaction of STIM1 with a new

${ }^{1}$ Department of Physiology (Cellular Physiology and Muscle Physiology Research Groups), University of Extremadura, 10003 Cáceres, Spain. ${ }^{2}$ Department of Medical Physiology and Biophysics, Institute of Biomedicine of Sevilla, Sevilla, Spain. Correspondence and requests for materials should be addressed to J.A.R. (email: jarosado@unex.es) 
a
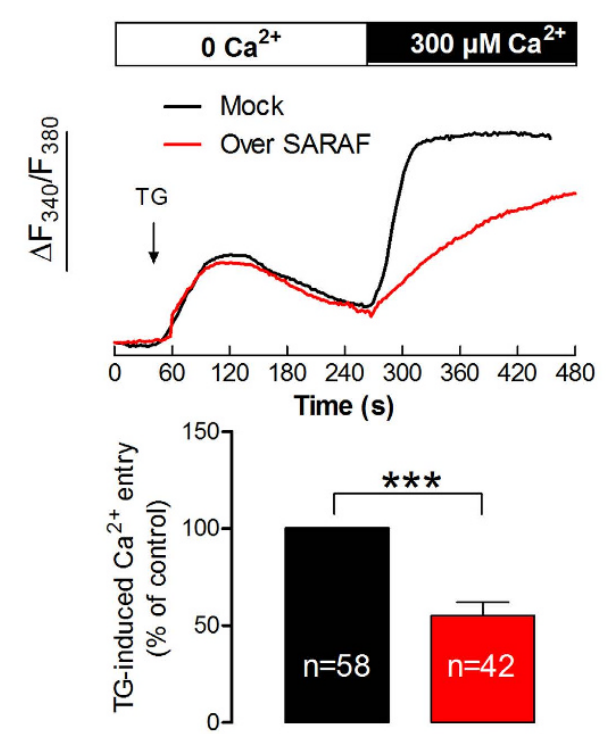

0

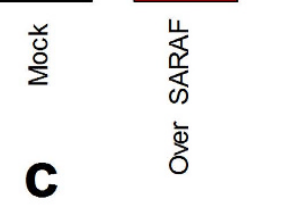

Blot: SARAF

Input: $\beta$-actin

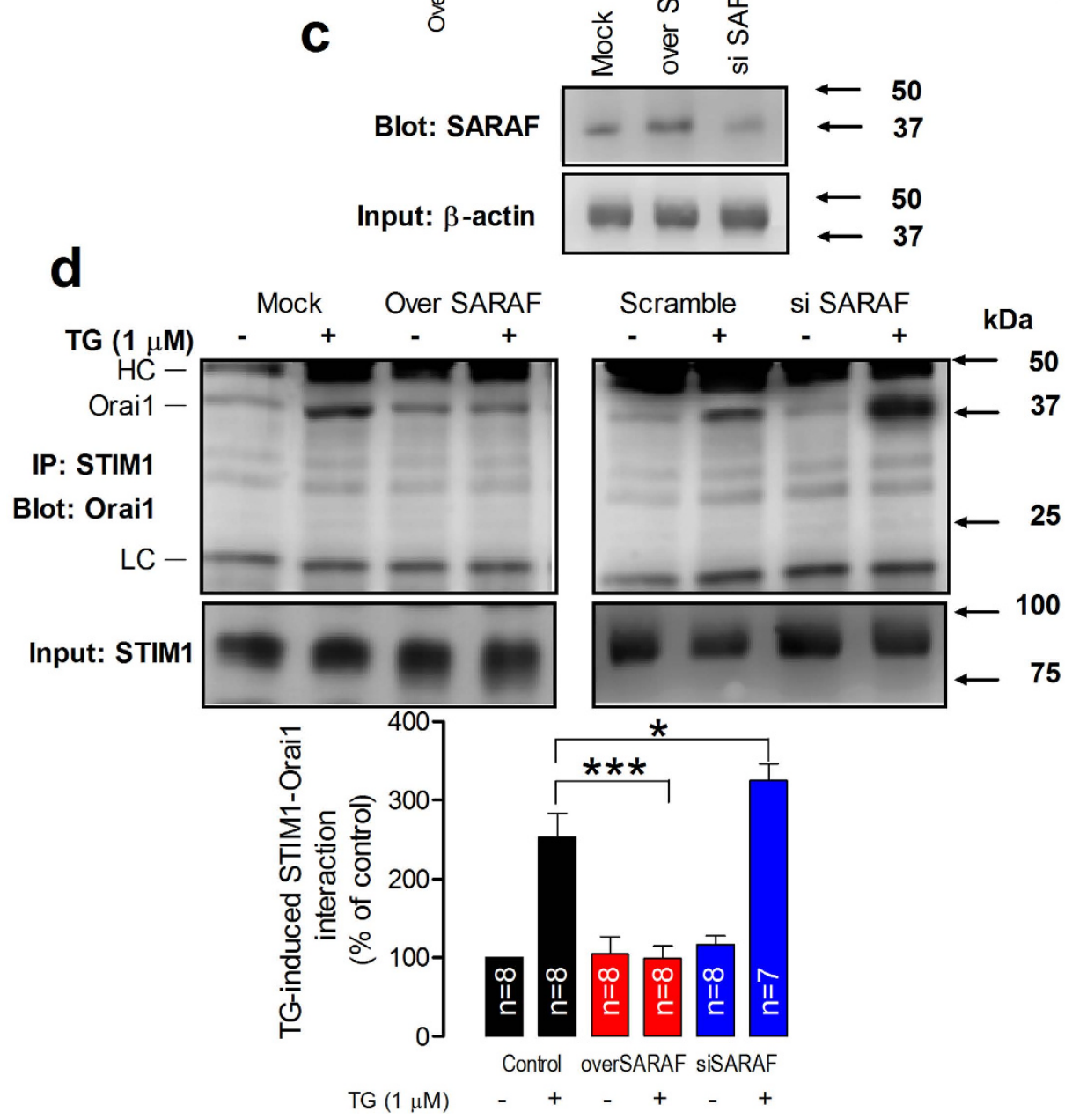

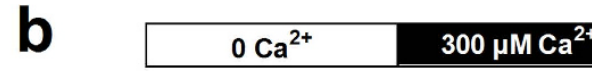
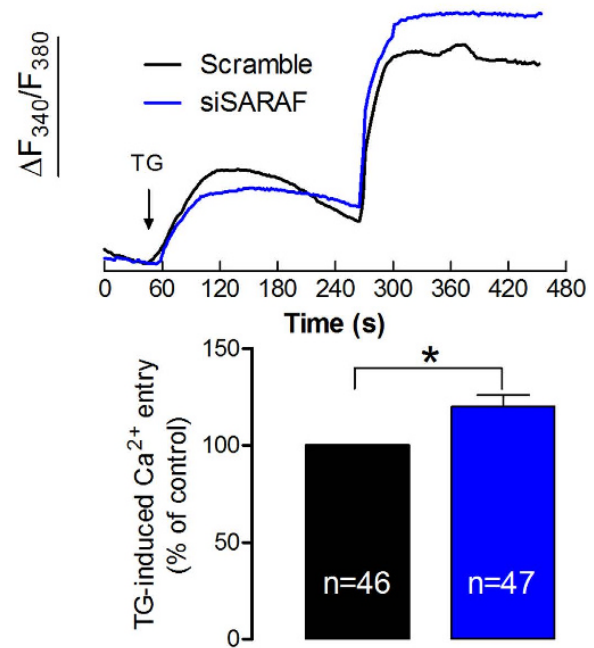

है

Figure 1. SARAF regulates TG-induced SOCE and STIM1-Orail interaction. (a,b) MEG-01 cells overexpressing SARAF and mock-treated cells (a) or MEG-01 cells transfected with si SARAF or scramble plasmid (b) were perfused with a $\mathrm{Ca}^{2+}$-free medium (100 $\mu \mathrm{M}$ EGTA added) and then stimulated with TG $(1 \mu \mathrm{M})$ followed by reintroduction of external Ca ${ }^{2+}$ (final concentration $300 \mu \mathrm{M}$ ) to initiate $\mathrm{Ca}^{2+}$ entry. Data are original traces representative of 42-58 experiments. Values are expressed as described in methods. The bar graph represents TG-induced $\mathrm{Ca}^{2+}$ entry as mean $\pm \mathrm{SEM}$ and data are presented as percentage of control. 
(c) Cells overexpressing SARAF, treated with siSARAF and mock-treated cells were lysed and subjected to Western blotting with anti-SARAF antibody, followed by reprobing with anti-actin antibody for protein loading control. (d) MEG-01 cells overexpressing SARAF or transfected with si SARAF and their respective controls were stimulated with TG $(1 \mu \mathrm{M})$ in a $\mathrm{Ca}^{2+}$-free medium $(100 \mu \mathrm{M}$ EGTA added) and three min later lysed. Whole cell lysates were immunoprecipitated (IP) with anti-STIM1 antibody and immunoprecipitates were subjected to $10 \%$ SDS-PAGE and subsequent Western blotting with a specific anti-Orail (aa 288-301 (Sigma)) antibody. Membranes were reprobed with the antibody used for immunoprecipitation for protein loading control. The panels show results from one experiment representative of 6-7 others. Molecular masses indicated on the right were determined using molecular-mass markers run in the same gel. HC, heavy chain of the antibody used for immunoprecipitation. The bar graph represents the quantification of STIM1-Orail association in resting and TG-treated cells. Results are recorded as arbitrary optical density units, expressed as mean \pm S.E.M. and presented as percentage of control. ${ }^{*}$ and ${ }^{* * *}$ represent $\mathrm{p}<0.05$ and $\mathrm{p}<0.001$, as compared to their respective controls.

regulatory protein named SARAF ${ }^{17}$. SARAF is a 339 -amino acid long protein with a putative single transmembrane domain and C-terminal serine/proline and arginine rich regions. SARAF is located in the membrane of the endoplasmic reticulum and interacts with the CTID region of STIM1 to mediate SCDI of Orail channels ${ }^{18}$. CTID has been described to have two functional lobes, STIM1(448-490) and STIM1(490-530), which cooperate to modulate the access of SARAF to the SOAR region, so that in the resting state, when the intracellular stores are filled with $\mathrm{Ca}^{2+}$, the CTID lobes facilitate access of SARAF to SOAR to keep SOAR in an inactive state, while store depletion results in an initial dissociation of SARAF, to allow activation of STIM1 and SOCE, followed by a reinteraction of SARAF with SOAR, facilitated by the STIM1(490-530) lobe, a process that has been associated with the SCDI of Orai $1^{17}$.

In the present study we have investigated the interaction of SARAF with STIM1 and Orail during the activation of SOCE. Our results indicate that SARAF is a modulator of the interaction between STIM1 and Orail channels that, during the initial steps of the activation of SOCE, transiently dissociates from STIM1 to associate with the $\mathrm{C}$-terminus of Orail to activate $\mathrm{Ca}^{2+}$ entry, and then reinteracts with STIM1 to mediate SCDI. These findings shed new light on the regulatory mechanism of SOCE.

\section{Results}

SARAF regulates store-operated $\mathrm{Ca}^{2+}$ entry and STIM1-Orai1 interaction. As depicted in Fig. 1a, treatment of MEG-01 cells with thapsigargin (TG) in a $\mathrm{Ca}^{2+}$-free medium resulted in a transient rise in cytosolic $\mathrm{Ca}^{2+}$ concentration $\left(\left[\mathrm{Ca}^{2+}\right]_{c}\right)$ due to passive $\mathrm{Ca}^{2+}$ release from the intracellular stores. The subsequent SOCE, upon readdition of $\mathrm{Ca}^{2+}$ to the medium, was significantly inhibited by $45 \%$ in cells overexpressing SARAF $(\mathrm{p}<0.001)$. As expected, SOCE was significantly enhanced by $20 \%$ in cells where endogenous SARAF levels were reduced by siRNA (Fig. 1b; $\mathrm{p}<0.05$ ).

To further explore the mechanism underlying the regulation of SOCE by SARAF we modified the expression of SARAF in MEG-01 cells endogenously expressing STIM1 and Orai $1^{19}$, as well as SARAF (Fig. 1c), and investigated the role of SARAF in the interaction between STIM1 and Orail. The experiments were performed in resting cells and upon stimulation with TG by looking for co-immunoprecipitation in whole cell lysates from cells overexpressing SARAF and SARAF-silenced cells. As shown in Fig. 1d, a detectable interaction between STIM1 and Orail was noticeable in resting cells, as previously described ${ }^{19}$. This interaction was significantly enhanced to $253 \pm 30 \%$ of control after $\mathrm{Ca}^{2+}$ store depletion. Overexpression of SARAF abolished TG-evoked interaction between STIM1 and Orail without having any significant effect on the resting interaction between both proteins. Consistent with this, SARAF silencing significantly enhanced the STIM1-Orail interaction induced by TG to $325 \pm 21 \%$ of control $(\mathrm{p}<0.05)$ and had a negligible effect on this interaction in resting cells. Analysis of the specificity of the anti-Orail and anti-STIM1 antibodies is depicted in the supplementary Fig. 1.

In an attempt to ascertain the role of SARAF in the STIM1-Orail association in $\mathrm{Ca}^{2+}$ store-depleted cells, we analyzed the interaction between Orail and the Orail-activating small fragment (OASF; amino acids 233-474) of STIM1 in NG115-401L cells, which express a negligible amount of endogenous STIM120,21. Cells were transfected with expression plasmids for pEYFP-Orail and pEYFP-OASF in combination with siSARAF or expression plasmid for SARAF. As shown in Fig. 2, co-expression of Orail and OASF resulted in a robust activation of $\mathrm{Ca}^{2+}$ entry independently of $\mathrm{Ca}^{2+}$ store depletion as compared to mock-treated cells, which did not display constitutive $\mathrm{Ca}^{2+}$ entry. $\mathrm{Ca}^{2+}$ entry induced by co-expression of Orail and OASF was impaired by overexpression of SARAF and enhanced by SARAF silencing (Fig. 2), thus suggesting that SARAF directly interferes with the interaction between the OASF region of STIM1 and Orail.

SARAF interacts with STIM1 and Orai1. It has been suggested that SARAF is associated with STIM1 in resting cells and upon store depletion undergoes dissociation of STIM1 followed by reinteraction to activate $\mathrm{SCDI}^{17}$; however, the functional role of SARAF when it is dissociated from STIM1 remains unknown. We have now further explored the temporal relationship of the association of SARAF with STIM1 and Orail in MEG-01 cells. We have explored the interaction of SARAF with STIM1 or Orail by in situ proximity ligation assay (PLA). Resting MEG-01 cells or cells stimulated with TG $(1 \mu \mathrm{M})$ for 30 or 120 s were subjected to PLA. As shown in Fig. 3 under resting conditions SARAF-Orail and SARAF-STIM1 interactions were detected in most cells analyzed. Treatment of MEG01 cells with TG $(1 \mu \mathrm{M})$ for $30 \mathrm{~s}$ enhanced the interaction SARAF-Orai1 as detected by the increased number of red dots, as compared to control. However, the number of associations between SARAF and STIM1 per cell was significantly reduced (and we were unable to detected them in a number of cells) 


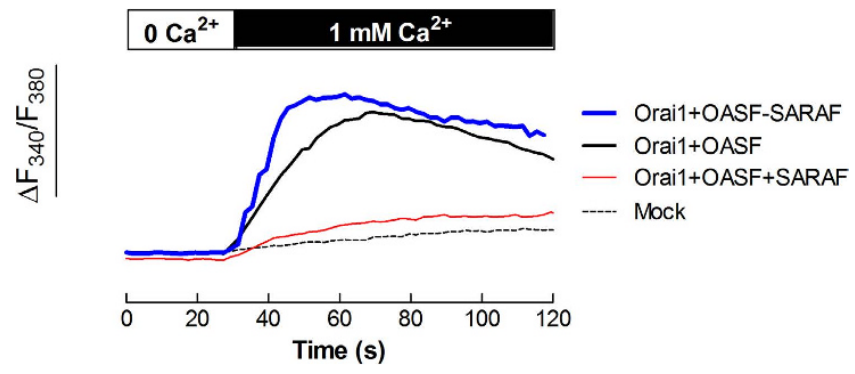

Figure 2. SARAF impairs OASF-dependent Orail function. NG115-401L cells were transfected with pEYPFOrail and pEYPF-OASF in the absence of presence of SARAF or siSARAF or were mock treated, as indicated. After $48 \mathrm{~h}$ cells were perfused with a $\mathrm{Ca}^{2+}$-free medium $(100 \mu \mathrm{M}$ EGTA added) followed by reintroduction of external $\mathrm{Ca}^{2+}$ (final concentration $1 \mathrm{mM}$ ) to initiate constitutive $\mathrm{Ca}^{2+}$ entry. Data are original traces representative of 42-58 experiments. Values are expressed as described in methods.

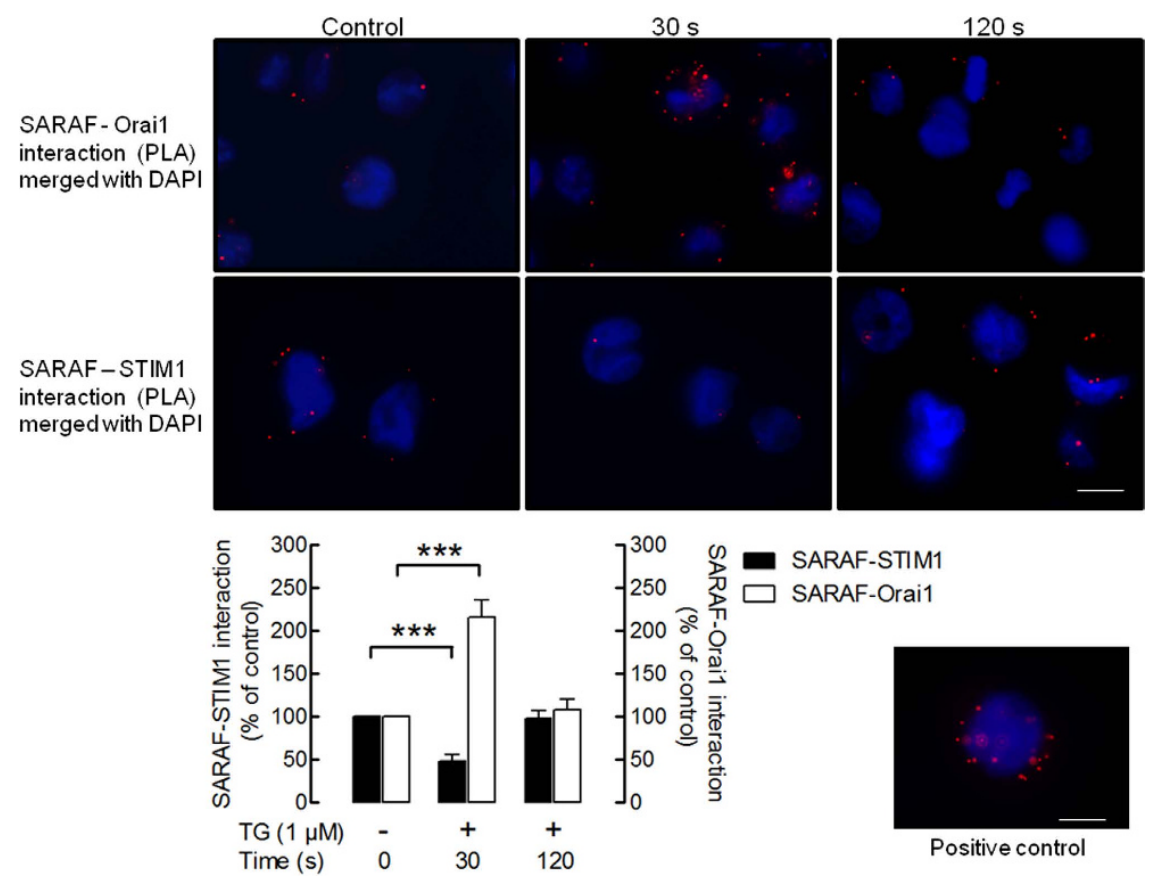

Figure 3. Dynamic interaction of SARAF with Orail and STIM1. MEG-01 cells were suspended in HBS containing $300 \mu \mathrm{M} \mathrm{CaCl}_{2}$ and were left untreated or stimulated with TG $(1 \mu \mathrm{M})$ for 30 or $120 \mathrm{~s}$. The cells were then fixed with $4 \%$ ice-cold paraformaldehyde and blocked using blocking solution from the Duolink In Situ Red kit ${ }^{\circledR}$ from Sigma. Samples were incubated with primary antibodies: rabbit anti-SARAF and mouse antiSTIM1 or mouse anti-Orail (SAB4200273, Sigma) antibodies. A Duolink assay was subsequently performed according to the manufacturer's instructions as described in Material and Methods. After performing the PLA procedure, images were taken with an Eclipse TE300 fluorescence microscope. Red spots represent individual dimers. The positive control is shown in the lower right corner. The bars indicate $10 \mu \mathrm{m}$. The bar graphs indicate association between SARAF and STIM1 or Orail expressed as the mean \pm S.E.M. of 100-200 cells. The quantification of PLA signals was performed using ImageJ software. ${ }^{\star * *} \mathrm{p}<0.001$.

upon stimulation for $30 \mathrm{~s}$ with TG. After $120 \mathrm{~s}$ of treatment with TG the number of red dots/cell representative of SARAF-Orail as well as SARAF-STIM1 interaction returned to levels similar to those observed in resting cells. The quantification of the PLA signal for the interaction between SARAF and STIM1 or SARAF and Orail revealed that treatment with TG enhanced the interaction between SARAF and Orail reaching a maximum of $215 \pm 21 \%$ of control (resting cells) after $30 \mathrm{~s}$ of stimulation and then returned to approximately the resting level after 120 s of treatment (Fig. 3; p < 0.001); Furthermore, TG significantly reduced the association between SARAF and STIM1 reaching a minimum of $48 \pm 7 \%$ of control after $30 \mathrm{~s}$ of stimulated and then increased, reaching the resting level at $120 \mathrm{~s}$ of treatment (Fig. 3; p < 0.001). These findings support the dynamic interaction of SARAF with STIM1 and Orail upon treatment with TG.

We further explored the interaction between SARAF and both STIM1 and Orail by co-immunoprecipitation. As shown in Fig. 4a, a detectable interaction between SARAF and both STIM1 and Orail was appreciable in resting cells. Treatment of MEG-01 cells with TG evoked a time-dependent effect on the association of SARAF 
a

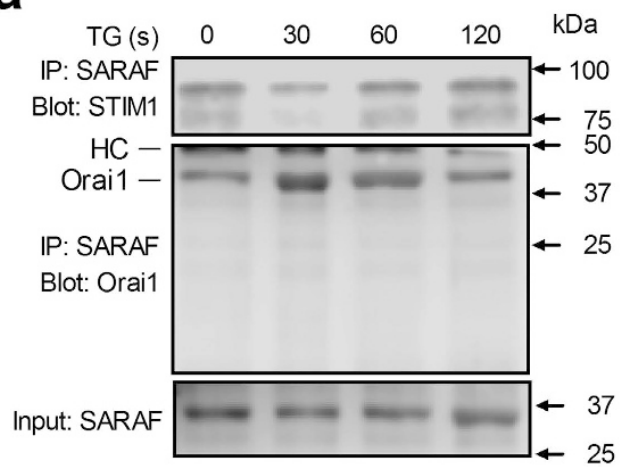

b
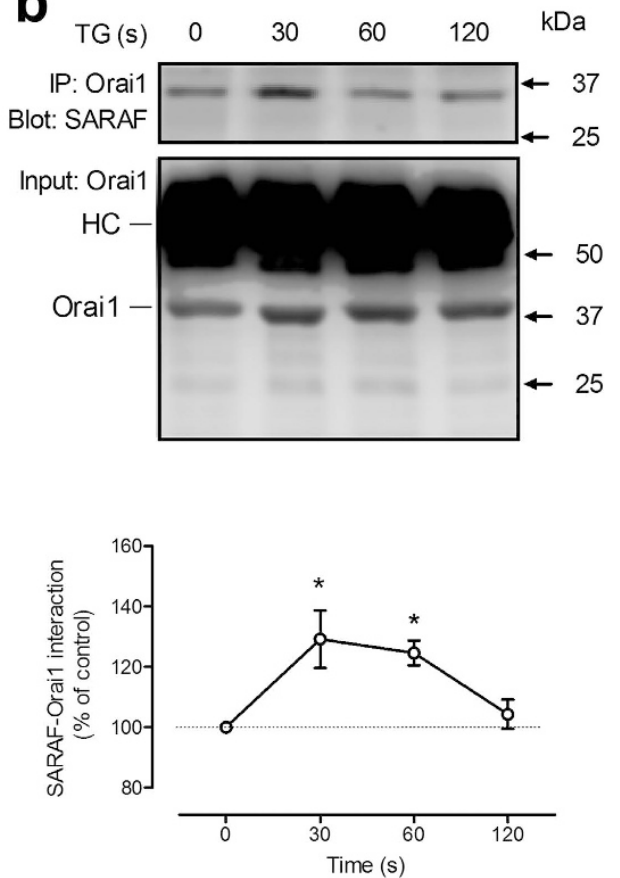

Figure 4. Time-course of TG-induced SARAF-STIM1 and SARAF-Orail co-immunoprecipitation. MEG01 cells (a) and NG115-401L cells (b) were stimulated with TG $(1 \mu \mathrm{M})$ in the presence of $300 \mu \mathrm{M}$ extracellular $\mathrm{Ca}^{2+}$. Samples were removed 30s before the addition of TG and 30, 60 and $120 \mathrm{~s}$ after the treatment with TG. Whole cell lysates were immunoprecipitated (IP) with the indicated antibody and immunoprecipitates were subjected to $10 \%$ SDS-PAGE and subsequent Western blotting with specific anti-Orail (aa 288-301 (Sigma)), anti-SARAF and anti-STIM1 antibodies. Membranes were reprobed with the antibody used for immunoprecipitation for protein loading control. The panels show results from one experiment representative of 5 others. Molecular masses indicated on the right were determined using molecular-mass markers run in the same gel. HC, heavy chain of the antibody used for immunoprecipitation. Data represent the quantification of SARAF-Orail and SARAF-STIM1 association in resting and TG-treated cells. Results are recorded as arbitrary optical density units, expressed as mean \pm S.E.M. and presented as percentage of control (resting cells). ${ }^{*}$ and ${ }^{* * *}$ represent $\mathrm{p}<0.05$ and $\mathrm{p}<0.001$, as compared to controls.

with STIM1 and Orai1. TG induced an initial significant decrease in SARAF-STIM1 interaction that reached a minimum of $35 \%$ of the resting level $30 \mathrm{~s}$ after stimulation $(\mathrm{p}<0.001)$. SARAF-STIM1 interaction then increased, exceeding basal levels approximately $120 \mathrm{~s}$ after stimulation. In parallel with these findings, treatment with TG significantly increased the association between SARAF and Orail reaching a maximum of $190 \%$ of the resting level $30 \mathrm{~s}$ after its addition $(\mathrm{p}<0.001)$ and then returned to the basal level at $120 \mathrm{~s}$ after stimulation (Fig. 4a).

In order to explore whether the interaction SARAF-Orail requires STIM1 we repeated the study in NG115401L cells lacking STIM1. As reported in Fig. 4b, our results indicate that, in the absence of STIM1, TG evokes association between SARAF and Orail, which reached a maximum $30 \mathrm{~s}$ after cell stimulation $(\mathrm{p}<0.001)$. These findings indicate that STIM1 is not responsible for the dissociation of the interaction between SARAF and Orail.

SARAF modulates $\mathrm{Ca}^{2+}$ entry through Orai1 independently of STIM1. To understand the functional significance of the interaction of SARAF with Orail independently of STIM1 we determined $\mathrm{Ca}^{2+}$ entry in NG115-401L cells transfected with expression plasmid for Orai1 in combination with SARAF overexpression or siSARAF. Since NG115-401L cells release $\mathrm{Ca}^{2+}$ in response to TG but do not display SOCE ${ }^{21}$, we used ATP, as a physiological agonist, to stimulate $\mathrm{Ca}^{2+}$ entry. As depicted in Fig. 5a, stimulation of NG115-401L cells with $100 \mu \mathrm{M}$ ATP in a Ca ${ }^{2+}$-free medium resulted in a transient rise in $\left[\mathrm{Ca}^{2+}\right]_{c}$ due to $\mathrm{Ca}^{2+}$ release from the intracellular stores. Overexpression of Orail alone or in combination with either SARAF silencing or overexpression did not significantly modify the ability of ATP to release $\mathrm{Ca}^{2+}$ from the stores. If we consider the entry of $\mathrm{Ca}^{2+}$ stimulated by ATP, Orail overexpression resulted in a significant increase in $\mathrm{Ca}^{2+}$ influx to $187 \pm 12 \%$ of control (Fig. 5b; $\mathrm{p}<0.05$ ), thus suggesting functional expression of Orail. SARAF silencing did not significantly altered ATP-induced $\mathrm{Ca}^{2+}$ entry per se but abolished the additional $\mathrm{Ca}^{2+}$ influx observed in cells overexpressing Orail (Fig. 5b,d; $\mathrm{p}<0.05$ ). By contrast, SARAF overexpression significantly increased ATP-stimulated $\mathrm{Ca}^{2+}$ influx in the wild type as well as in the Orail-overexpressing NG115-401L cells (Fig. 5b,d; $p<0.05$ ). Figure. 5c shows the amount of $\mathrm{Ca}^{2+}$ accumulated in the intracellular $\mathrm{Ca}^{2+}$ stores, estimated by organelle content release using ionomycin $(2 \mu \mathrm{M})$. As depicted in Fig. 5c, Orail expressing did not significantly alter the ability of NG115-401L cells to accumulate $\mathrm{Ca}^{2+}$ into the stores but SARAF silencing significantly enhanced the amount of $\mathrm{Ca}^{2+}$ released using 
a

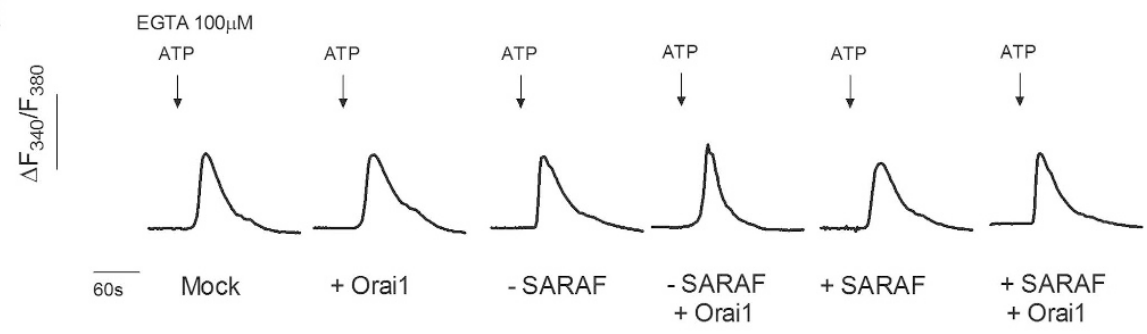

b

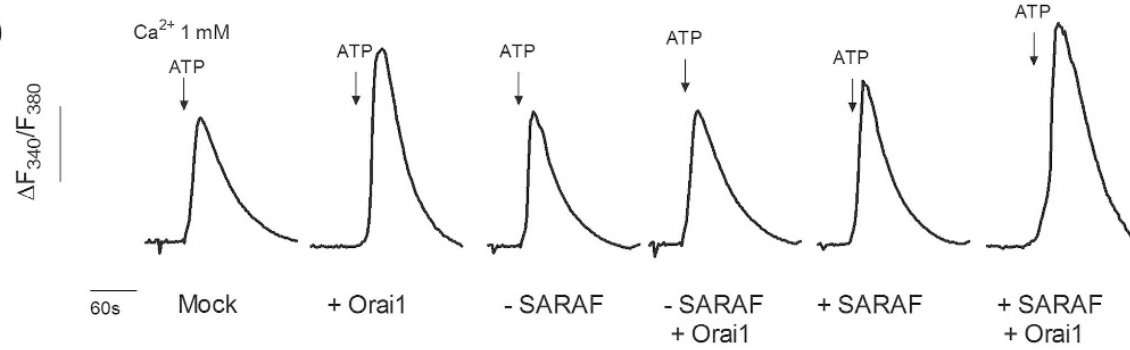

C

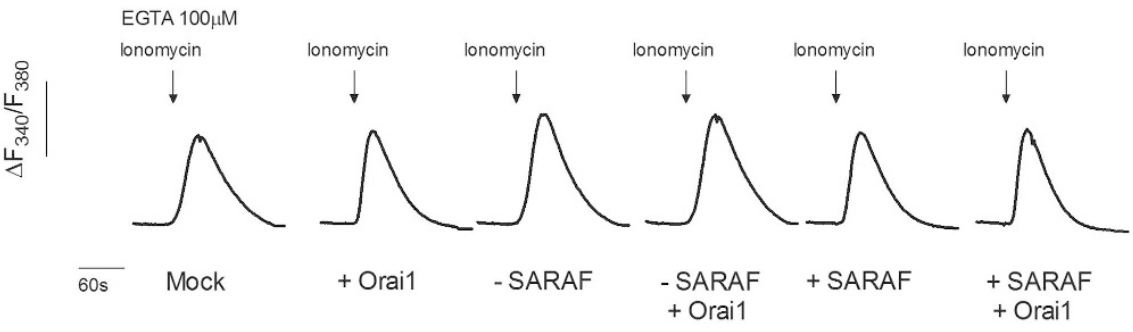

d

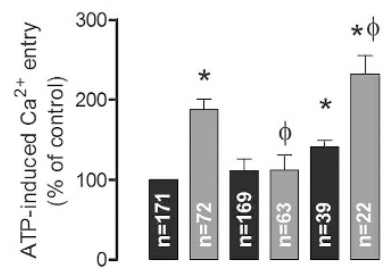

overexpression Orai1 - +-++

overexpression SARAF - $-\quad-\quad++$

Si SARAF - -++-

e

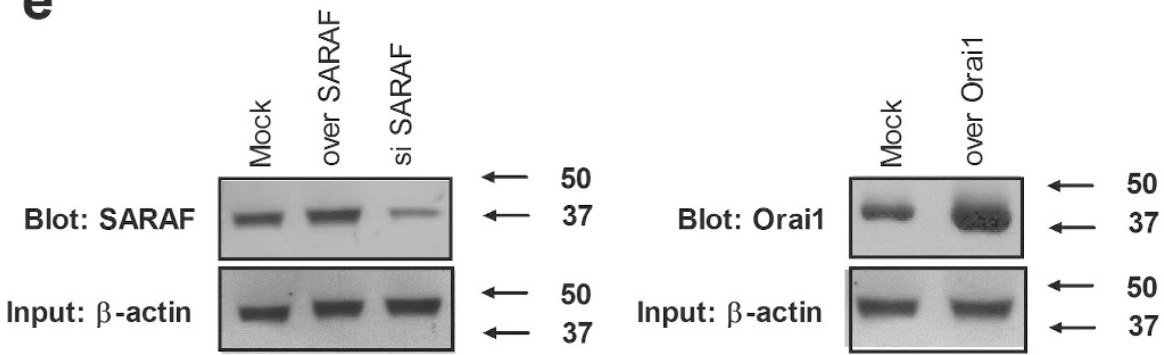

Figure 5. SARAF regulates Orail channel function. (a-c) NG115-401L cells were transfected with Orail, SARAF, siSARAF or empty vector as control (Mock). Cells were perfused with a $\mathrm{Ca}^{2+}$-free medium $(100 \mu \mathrm{M}$ EGTA added) $(\mathbf{a}, \mathbf{c})$ or a medium containing $1 \mathrm{mM} \mathrm{Ca}^{2+}(\mathbf{b})$ and then stimulated with ATP $(100 \mu \mathrm{M} ;(\mathbf{a}, \mathbf{b}))$ or ionomycin $(2 \mu \mathrm{M},(\mathbf{c}))$. Data are original traces representative of 22-171 experiments. (d) The bar graph represents ATP-evoked $\mathrm{Ca}^{2+}$ entry estimated as the integral of the rise in $\left[\mathrm{Ca}^{2+}\right]_{c}$ above basal for $1 \frac{1 / 2}{2}$ min after the addition of ATP in the presence of external $\mathrm{Ca}^{2+}$, corrected by subtraction of the integral over the same period for stimulation with ATP in the absence of external $\mathrm{Ca}^{2+}$. Data are expressed as mean $\pm \mathrm{SEM}$ and presented as percentage of control (mock-treated cells). ${ }^{*}$ and ${ }^{\phi}$ represent $\mathrm{p}<0.05$ as compared to ATP-induced $\mathrm{Ca}^{2+}$ entry in mock-treated controls or cells overexpressing Orail, respectively. (e) Immunoblot analysis of SARAF and Orail expressed in NG115-401L cells before or after treatment with siSARAF or expression plasmids for SARAF and Orail using anti-SARAF antibody and anti-Orail antibody (aa 288-301 (Sigma)). Membranes were reprobed with anti- $\beta$-actin antibody for protein loading control. The panel shows results from one experiment representative of 3 others. Molecular masses indicated on the right were determined using molecular-mass markers run in the same gel. 

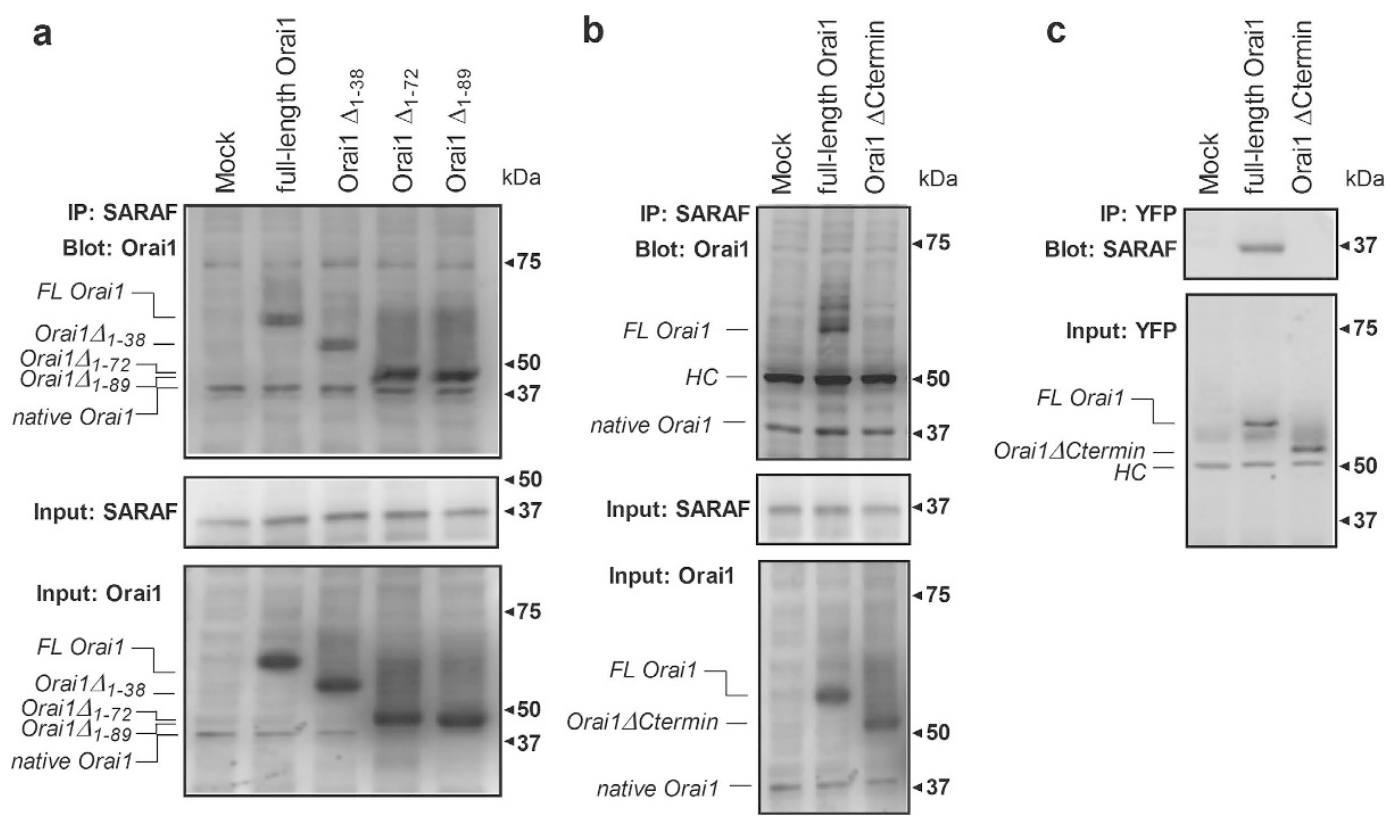

Figure 6. Analysis of the Orail site that interacts with SARAF. NG115-401L cells were transfected with SARAF alone or in combination with either pEYFP-full-length Orail (FL-Orai1), the N-terminal deletion mutants (pEYFP-Orail $\Delta \mathrm{N}_{1-38}\left(\right.$ Orail $\left.\Delta N_{1-38}\right), \Delta \mathrm{N}_{1-72}\left(\right.$ Orail $\left.\Delta N_{1-72}\right)$ and $\Delta \mathrm{N}_{1-89}\left(\right.$ Orai1 $\left.\Delta N_{1-89}\right)($ a), pEYFPOrail C-terminal deletion mutant (Orai1- $\Delta$ Ctermin, amino acids 1-260 (b,c) or empty vector (Mock), as indicated. After $48 \mathrm{~h}$ cells were lysed and the whole cell lysates were immunoprecipitated (IP) with anti-SARAF antibody (a,b) or anti-YFP antibody (c). Immunoprecipitates were subjected to 10\% SDS-PAGE and subsequent Western blotting with a specific anti-Orai1 (amino acids 288-301, Sigma) antibody (a), anti-Orail (ab177021, Abcam) antibody (b) or anti-SARAF antibody (c). Membranes were reprobed with the antibody used for immunoprecipitation for protein loading control (middle panels). (a,b) Alternatively, the cell lysates were subjected to $10 \%$ SDS-PAGE and subsequent Western blotting with anti-Orail antibody (specific for amino acids 288-301; panel a, bottom) or with anti-Orail (ab177021, Abcam; panel b, bottom). HC, heavy chain of the antibody used for immunoprecipitation. The panels show results from one experiment representative of 3 others. Molecular masses indicated on the right were determined using molecular-mass markers run in the same gel.

ionomycin by $20 \%(\mathrm{p}<0.05)$ as previously described in HeLa and Jurkat cells ${ }^{18}$. Figure $5 \mathrm{e}$, shows the expression of SARAF and Orail proteins in cells treated as mentioned above. These findings indicate that SARAF plays a positive regulatory role on Orail channel function, and, together with the data presented in Figs 2-4, provide evidence for the first time of a direct and functional interaction of SARAF with Orail.

Blockade of the C-terminal region of Orai1 displays a significant reduction in SARAF binding. Finally, we have investigated the location of the possible SARAF binding site of Orail in NG115-401L cells overexpressing pEYFP-Orail (full-length), the N-terminal deletion mutants (pEYFP-Orail $\Delta \mathrm{N}_{1-38}, \Delta \mathrm{N}_{1-72}$ and $\Delta \mathrm{N}_{1-89}$ ) or the C-terminal deletion mutant (Orai1- $\Delta$ Ctermin, amino acids 1-260) together with SARAF. As shown in Fig. 6a, our results indicate that co-expression of pEYFP-Orail and SARAF show detectable co-immunoprecipitation between both proteins, which was not altered in cells co-transfected with SARAF and the $\mathrm{N}$-terminal truncations of Orail described above, which strongly indicates that the cytosolic N-terminal region of Orail is not required for SARAF interaction. By contrast, we were unable to detect co-immunoprecipitation between SARAF and the Orail- $\Delta$ Ctermin (Fig. $6 \mathrm{~b}, \mathrm{c}$ ), thus suggesting that SARAF interacts with the C-terminus of Orail.

To further assess the relevance of the C-terminal region of Orail in the interaction with SARAF we used a manoeuvre based on the blockade of this region by introduction of an antibody that specifically recognizes the sequence 288-301 of Orail (anti-OrailCT, Sigma) as compared to the introduction of an antibody that recognizes 18 amino acids located in the N-terminal region (anti-OrailNT, Prosci). The presence of these antibodies inside the cells was investigated by immunoprecipitation without adding any additional anti-Orail antibody and subsequent Western blotting with the anti-Orail antibody $(288-301 \text {, Sigma })^{22}$. As shown in the supplemental Fig. 2a, first lane, Orail was clearly detected in cells that had been previously transfected with anti-Orai1CT or anti-Orai1NT antibodies. A faint band was detected when the Western blot was performed in the absence of primary antibody (primary-free control; supplemental Fig. 2a second lane), which reveals that the band detected by Western blotting using anti-Orail antibody was specifically Orail. Cells transfection with anti-OrailCT significantly attenuated the interaction of Orail with SARAF as compared to cells transfected with anti-Orai1NT or non-specific rabbit IgG (supplemental Fig. 2b,c). The lack of effect of transfection with anti-OrailNT confirms the 
results obtained using different $\mathrm{N}$-terminal deletion mutants. These findings indicate that SARAF interacts with a site located in the C-terminus of Orail.

\section{Discussion}

The present study provides relevant information concerning the mechanism of SOCE regulation by SARAF. We have found for the first time that SARAF associates with Orail and enhances Orail function in STIM1-deficient cells. SARAF interacts with the C-terminal region of Orail since Orail constructs lacking the C-terminus are unable to associate with SARAF and blockade of this region also impairs its ability to interact with SARAF. Our results suggest that SARAF dissociates from STIM1 upon $\mathrm{Ca}^{2+}$ store discharge probably to interact with Orai1 and cooperate with STIM1 in the activation of Orail and, then SARAF rapidly re-associates with STIM1 (and dissociates from Orai1) to participate in the inactivation of SOCE. Our results indicate that SARAF interacts and activates Orail channels in a scenario that allows direct association of both proteins independently of STIM1, which has been reported to be negative regulated by SARAF ${ }^{17,18}$.

Current evidence indicates that the Orail forming channels (i.e. CRAC, arachidonic acid-regulated $\mathrm{Ca}^{2+}$ and leukotriene $\mathrm{C} 4$-regulated $\mathrm{Ca}^{2+}$ channels ${ }^{23-25}$ ) are mostly regulated by STIM1. Similarly, there is a body of evidence for a role of STIM1 in the regulation of TRP channels as components of store-operated $\mathrm{Ca}^{2+}$ (SOC) channels, also involving Orail subunits ${ }^{22,26-30}$, Interestingly, Jha and coworkers have reported that, while in the resting state SARAF binds to SOAR in STIM1 (a process regulated by the CTID lobes), activation of Orail after $\mathrm{Ca}^{2+}$ store depletion requires dissociation of SARAF to allow SOAR to interact with and activate Orail channels. Subsequently, SARAF associates again with STIM1 to mediate SCDI. These findings are based on the observation that Orail current activated by the constitutively active STIM1(4E/4A) and STIM1(D76A) mutants was unaffected by SARAF, as well as on the role of different STIM1 mutants on SCDI of Orail currents ${ }^{17}$. In agreement with this study, we have found that SARAF, which co-immunoprecipitates with STIM1 under resting conditions, dissociates from STIM1 upon $\mathrm{Ca}^{2+}$ store depletion reaching a minimum after 30 s of stimulation and then reinteracts with STIM1, and opposite results were observed when we tested the interaction of SARAF with Orail, revealing that 30 s after the initiation of $\mathrm{Ca}^{2+}$ store depletion there is an increase in SARAF-Orail co-immunoprecipitation followed by a decrease in the association between both proteins. Using STIM1-deficient NG115-401L cells we have found that dissociation of SARAF and Orail observed $30 \mathrm{~s}$ after treatment with TG occurs independently of STIM1. The dynamic interactions of SARAF with STIM1 or Orail were found to occur with the same time courses, which might suggest that SARAF dissociates from STIM1 in the initial stages of $\mathrm{Ca}^{2+}$ store depletion to interact with Orail, although the mechanism underlying this translocation is unclear at this stage.

The interaction of SARAF with Orail might transiently cooperate with STIM1 in the activation of Orail currents. However, the predominant role of SARAF on SOCE is the negative regulation of $\mathrm{Ca}^{2+}$ entry via activation of SCDI as evidenced by us in manoeuvres modifying the expression of SARAF in MEG01 cells endogenously expressing STIM1 and Orail or by other authors in different cell types ${ }^{17,18,31}$, where SARAF overexpression or silencing SARAF expression leads to attenuated or enhanced SOCE, respectively. The lack of observation of a positive role of SARAF on the initial stages of SOCE suggests that the transient stimulatory role of SARAF on the activation of Orail might be a redundant function in the presence of functional STIM1, which might mask this effect and evidence solely the long lasting negative regulatory role of SARAF underlying the activation of SCDI. Alternatively, the lack of detection of a transient positive effect of SARAF overexpression in the initial stages of SOCE might be attributed to a negative regulation of other STIM1-regulated channels involved in SOCE by

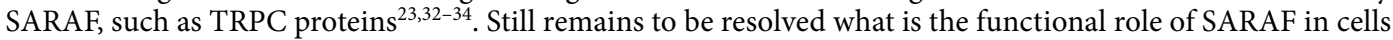
with a low expression of STIM1 but with a significant expression of Orail and other $\mathrm{Ca}^{2+}$ permeable channels. Our results indicate that, in cells with a low expression of STIM1, SARAF enhances Orail function, which might play a positive role in $\mathrm{Ca}^{2+}$ entry through store-independent mechanisms. Unveiling the role of SARAF on TRP channel function in cells with a low STIM1 expression might provide relevant information concerning the role of SARAF on store-independent $\mathrm{Ca}^{2+}$ entry.

In summary, on the base of the previous studies ${ }^{17,18}$ and our findings, we propose a model where SARAF modulates Orail channel function through direct and indirect interaction. At rest, SARAF is associated with the SOAR/OASF region of STIM1 via the CTID domain; however, upon $\mathrm{Ca}^{2+}$ store depletion SARAF transiently dissociates from STIM1 and associates with the C-terminal region of Orail. A few seconds later, and probably mediated by the increase in $\left[\mathrm{Ca}^{2+}\right]_{\mathrm{c}}$, SARAF dissociates from Orail and reinteracts with STIM1. Dissociation of SARAF from Orail, together with the subsequent association with STIM1 might have a role in the SCDI of Orail.

\section{Methods}

Materials. Fura-2 acetoxymethyl ester (fura-2/AM), was from Molecular Probes (Leiden, The Netherlands). Thapsigargin (TG), ATP, rabbit polyclonal anti-Orail antibody (catalog number O8264, epitope: amino acids 288-301 of human Orai1), Duolink ${ }^{\circledR}$ In Situ Red Starter Kit Mouse/Rabbit, mouse monoclonal anti-Orail antibody (clone ORAI1-89, epitope: synthetic peptide located near the C-terminus of human Orail, catalog number: SAB4200273), rabbit polyclonal anti- $\beta$-actin antibody (catalog number A2066, epitope: amino acids 365-375 of human $\beta$-actin), and bovine serum albumin (BSA) were from Sigma (Madrid, Spain). Rabbit polyclonal antiTMEM66 (SARAF) antibody (catalog number PA5-24237. epitope: amino acids 33-62 of the N-terminal region of human TMEM66) and Turbofect transfection reagent were from Thermo Fisher (Madrid, Spain). Mouse monoclonal anti STIM1 antibody (Clone 44/GOK, epitope: amino acids 25-139 of human STIM1, catalog number 610954) was from BD Transduction Laboratories (Frankin Lakes, NJ, U.S.A.). Calmidazolium chloride was from Bio-Rad (Madrid, Spain). Rabbit polyclonal anti-Orail antibody (catalog number: ab177021, epitope: peptide from the extracellular loop 2 region), horseradish peroxidase-conjugated anti-mouse IgG antibody and 
anti-rabbit IgG antibody for IP (not recognizing the heavy and light chains of the immunoprecipitating antibody) were from Abcam (Madrid, Spain). Rabbit polyclonal anti-Orail antibody (catalog number: 4041, epitope: raised against an 18 amino acid synthetic peptide from near the N-terminus of human Orail) was from Prosci (Fort Collins, CO, USA). Amaxa nucleofector kit $\mathrm{C}$ was purchased from (Lonza, Madrid, Spain). Protein A-agarose was from Upstate Biotechnology Inc. (Madrid, Spain). Mouse anti-GFP antibody (mixture of two monoclonal antibodies, clones 7.1 and 13.1, which recognize both wild-type and mutant forms of GFP, such as YFP ${ }^{35}$; catalogue number 11814460001) and complete EDTA-free protease inhibitor tablets were from Roche (Madrid, Spain). Enhanced chemiluminescence detection reagents were from Pierce (Cheshire, U. K.). TransPass P was from New England Biolabs (Ipswich, MA, U.S.A.). Taq Polimerase, T4 ligase, EcoRI and EcoRV enzymes were from Takara (Bio Inc, Japan). Ultraclean GelSpin Kit ${ }^{\circledR}$ and Ultraclean Midi plasmid prep Kit ${ }^{\circledR}$ were from MoBio (MO BIO Laboratories, Carlsbad, USA). All other reagents were of analytical grade.

Plasmids construction. Plasmids were based on the previously published SARAF sequences (GenBank: JQ348891.1). The DNA of the complete cds was isolated from NG115-401L cells using specific primers (Forward: 5' AAAAAACCCGGGATGGCCGCAGCCTGCGGGCC 3'; and reverse: 5'AAAAAAGAATTCTTATCGTCTCCTGGTACCACCATAT-3'). Final cDNA was purified and cloned into the EcoRV site previously inserted in the pIRES2-eGFP-RV expression vector. Nucleotide sequence of this construct was verified by sequencing.

To knockdown expression of SARAF1, a pLKO.1-puro plasmid-based shRNA targeting the sequence: CGGACTTAGATATTGCATACA (clone ID: TRCN0000146643; Sigma-Aldrich) was used (SARAF-shRNA). In addition, a non-targeting shRNA plasmid (NT-shRNA) that targets no known human sequence was used as a control. A primer containing the target sequence along with a stem loop followed by the reverse target sequence was annealed to a complimentary primer and inserted into the EcoRI and AgeI sites of the pLKO.1-puro plasmid (Addgene; number 10878). The resulting hairpin consisted of the following sequence: $5^{\prime}$-CCGGCGGACTTAGATATTGCATACACTCGAGTGTATGCAATATCTAAGTCCGTTTTTTG- ${ }^{\prime}$. The correct insertion of the hairpin into pLKO.1 plasmid was finally checked by sequencing.

Cell culture and transfection. The megakaryoblastic cell line MEG01 was used in this study as a cellular model endogenously expressing STIM1. Moreover, STIM1-deficient NG115-401L cells were used for certain experimental procedures to avoid interference with endogenous STIM1. MEG01 and NG115-401L cell lines were obtained from ATCC (Manassas, VA, USA) and Sigma (Madrid, Spain), respectively, and cultured at $37^{\circ} \mathrm{C}$ with a $5 \% \mathrm{CO}_{2}$ in RPMI or DMEM, respectively, supplemented with $10 \%(\mathrm{v} / \mathrm{v})$ fetal bovine serum, $2 \mathrm{mM} \mathrm{L}$-glutamine and $100 \mathrm{U} / \mathrm{mL}$ penicillin and streptomycin.

Cells were transfected with expression plasmids for pIRES2-EGFP-SARAF, pEYFP-Orail, pEYPF-OASF, the pEYFP-Orail $\mathrm{N}$-terminal deletion mutants (Orail $\Delta \mathrm{N}_{1-38}, \Delta \mathrm{N}_{1-72}$ and $\Delta \mathrm{N}_{1-89}$ ) or the pEYFP-Orail C-terminal deletion mutant (Orail- $\Delta$ Ctermin, amino acids 1-260) kindly provided by Dr. Romanin, as well as with the siSARAF or scramble plasmid as described previously ${ }^{36-38}$ using Amaxa nucleofector technology (for MEG-01 cells) or Turbofect transfection reagent (for NG115-401L cells) and were used $48 \mathrm{~h}$ after transfection. For transfection with antibodies we used transpass $\mathrm{P}$ following the manufacturer's instructions and were used $6 \mathrm{~h}$ after transfection.

Cell transfection with shOrail was performed as described previously ${ }^{39}$. For the shOrail, the sense sequence was $5^{\prime}$-CACCTCACTGGTTAGCCATAAGACGAATCTTATGGCTAACCAGTGA-3' , and the antisense sequence was $5^{\prime}$-AAAACCTTTACACGCTAGATGGTTTGCTCTTATGGCTAACCAGTGA-3' ${ }^{\prime}$. Plasmids were used for silencing experiments at $1 \mu \mathrm{g} / \mu \mathrm{l}$.

Measurement of cytosolic free-calcium concentration $\left(\left[\mathrm{Ca}^{2+}\right]_{c}\right)$. Cells were loaded with fura-2 by incubation with $2 \mu \mathrm{M}$ fura $2 / \mathrm{AM}$ for $30 \mathrm{~min}$ at room temperature. Coverslips with cultured cells were mounted on a perfusion chamber and placed on the stage of an epifluorescence inverted microscope (Nikon Diaphot T200, Melville, NY, USA) with image acquisition and analysis system for videomicroscopy (Hamamatsu Photonics, Hamamatsu, Japan). Cells were continuously superfused with HEPES-buffered saline (HBS) containing (in mM): $125 \mathrm{NaCl}, 5 \mathrm{KCl}, 1 \mathrm{MgCl}$, 5 glucose, $25 \mathrm{HEPES}$, and $\mathrm{pH} 7.3$, supplemented with $0.1 \%$ (w/v) BSA.. Cells were alternatively excited with light from a xenon lamp passed through a high-speed monochromator (Polychrome IV, Photonics, Hamamatsu, Japan) at $340 / 380 \mathrm{~nm}$. Fluorescence emission at $505 \mathrm{~nm}$ was detected using a cooled digital CCD camera (Hisca CCD C-6790, Hamamatsu, Japan) and recorded using Aquacosmos 2.5 software (Hamamatsu Photonics, Hamamatsu, Japan). Fluorescence ratio $\left(\mathrm{F}_{340} / \mathrm{F}_{380}\right)$ was calculated pixel by pixel and data are presented as $\Delta \mathrm{F}_{340} / \mathrm{F}_{380}$. ATP-evoked $\mathrm{Ca}^{2+}$ influx was measured as the integral of the rise in $\left[\mathrm{Ca}^{2+}\right]_{\mathrm{c}}$ above basal for $1 \frac{1 / 2}{2}$ in after the addition of ATP in the presence of external $\mathrm{Ca}^{2+}$, corrected by subtraction of the integral over the same period for stimulation in the absence of external $\mathrm{Ca}^{2+}$ (with $100 \mu \mathrm{M}$ EGTA).

Immunoprecipitation and Western blotting. The immunoprecipitation and Western blotting were performed as described previously ${ }^{40}$. Briefly, $500 \mu \mathrm{L}$ aliquots of cell suspension $\left(4 \times 10^{6} \mathrm{cell} / \mathrm{mL}\right)$ were lysed with an equal volume of ice-cold $2 \times \mathrm{NP}-40$ buffer, $\mathrm{pH} 8$, containing $274 \mathrm{mM} \mathrm{NaCl}, 40 \mathrm{mM}$ Tris, $4 \mathrm{mM} \mathrm{EDTA}, 20 \%$ glycerol, $2 \%$ nonidet P-40, $2 \mathrm{mM} \mathrm{Na}_{3} \mathrm{VO}_{4}$ and complete EDTA-free protease inhibitor tablets. Aliquots of cell lysates $(1 \mathrm{~mL})$ were immunoprecipitated by incubation with $1 \mu \mathrm{g}$ of anti-STIM1 or $2 \mu \mathrm{g}$ of anti-SARAF or anti-Orail antibody and $25 \mu \mathrm{L}$ of protein $\mathrm{A}$-agarose overnight at $4{ }^{\circ} \mathrm{C}$ on a rocking platform. The immunoprecipitates were resolved by $8 \%$ SDS-PAGE and separated proteins were electrophoretically transferred onto nitrocellulose membranes for subsequent probing. Blots were incubated overnight with $10 \%(\mathrm{w} / \mathrm{v}) \mathrm{BSA}$ in tris-buffered saline with $0.1 \%$ Tween 20 (TBST) to block residual protein binding sites. Immunodetection of Orail, STIM1 and SARAF 
was achieved by incubation for $2 \mathrm{~h}$ with anti-STIM1 antibody diluted 1:250 in TBST, anti-Orail antibody diluted 1:200 in TBST, the anti-SARAF antibody diluted 1:1000 in TBST and anti-calmodulin antibody diluted 1:500. The primary antibody was removed and blots were washed six times for 5 min each with TBST. To detect the primary antibody, blots were incubated for $1 \mathrm{~h}$ with horseradish peroxidase-conjugated goat anti-mouse IgG antibody or horseradish peroxidase-conjugated goat anti-rabbit IgG antibody diluted 1:10000 in TBST and then exposed to enhanced chemiluminiscence reagents for $4 \mathrm{~min}$. The density of bands was measured using C-DiGit Chemiluminescent Western Blot Scanner. Data were normalized to the amount of protein recovered by the antibody used for the immunoprecipitation.

Proximity ligation assay. The PLA was performed using the Duolink ${ }^{\circledR}$ In Situ Red Starter Kit Mouse/ Rabbit and performed following the manufacturer's instructions and as previously described ${ }^{41}$. After stimulation, the cells were fixed in ice-cold 4\% paraformaldehyde in phosphate-buffered saline (PBS) for $10 \mathrm{~min}$. The cells were then washed three times with PBS with agitation and permeabilized in PBS that contained $0.5 \%$ Triton $\mathrm{X}-100$ for $10 \mathrm{~min}$ at room temperature, followed by washing the cells twice with $0.05 \%$ Tween 20 in Tris-buffered saline buffer (TBS, containing in $\mathrm{mM}$ : 50 Tris- $\mathrm{HCl}[\mathrm{pH} 7.5]$ and $150 \mathrm{NaCl}$ ). Cells were then blocked for $30 \mathrm{~min}$ with one drop of Duolink Blocking solution in a humidified chamber at $37^{\circ} \mathrm{C}$ and incubated for $1 \mathrm{~h}$ at $37^{\circ} \mathrm{C}$ with appropriate combinations of antibodies diluted 1:100 in Duolink Antibody Diluent solution $(40 \mu \mathrm{L})$. The antibodies used for the PLA were rabbit anti-TMEM66 (SARAF) (Thermo Fisher, Madrid, Spain) combined with mouse anti-Orail (Sigma, Madrid, Spain) or mouse anti-STIM1 (BD Transduction Laboratories, Frankin Lakes, NJ, U.S.A.). After washing with Wash Buffer A, the cells were incubated for $1 \mathrm{~h}$ at $37^{\circ} \mathrm{C}$ with PLA probes, which are secondary antibodies (Duolink ${ }^{\circledR}$ In Situ PLA Probe anti-rabbit PLUS or Duolink ${ }^{\circledR}$ In Situ PLA Probe anti-mouse MINUS) conjugated to unique oligonucleotides. The cultures were further subjected to in situ PLA using a Duolink Detection kit according to the manufacturer's instructions. Briefly, the slides were incubated with Ligation-Ligase solution in a pre-heated humidified chamber for $30 \mathrm{~min}$ at $37^{\circ} \mathrm{C}$, followed by incubation with amplification polymerase solution for an additional $100 \mathrm{~min}$ at $37^{\circ} \mathrm{C}$. Finally, the cells were washed in Wash Buffer B, and the slides were mounted using Mounting Medium with DAPI ${ }^{\circledR}$ and evaluated using a Eclipse TE300 fluorescence microscope with a $\times 60$ oil-immersion objective (Nikon Corporation, Tokyo, Japan). The quantification of PLA signals was performed using ImageJ software (NIH, U.S.A.). The processed images were thresholded and the in situ PLA signals per cell were quantified. The settings were kept constant for all of the images throughout the experiments. Quantifications were performed from 30 to 40 images from a minimum of four slides for each treatment corresponding to 100-200 cells. Mouse anti-Orail directed towards the C-terminus (catalog number SAB4200273, Sigma) combined with rabbit anti-Orail directed towards 18 amino acids located in the N-terminal region of human Orail (catalog number 4041, Prosci) was used as a positive control. As a negative technical control, the primary antibodies were used alone, or both primary antibodies were omitted. The negative controls did not yield any significant PLA signals at any treatment condition.

Statistical Analysis. Analysis of statistical significance was performed using one-way analysis of variance. For comparison between two groups Student's $t$ test was used. $P<0.05$ was considered to be significant for a difference.

\section{References}

1. Roos, J. et al. STIM1, an essential and conserved component of store-operated $\mathrm{Ca}^{2+}$ channel function. J Cell Biol 169, 435-445 (2005).

2. Liou, J. et al. STIM is a Ca ${ }^{2+}$ sensor essential for $\mathrm{Ca}^{2+}$-store-depletion-triggered $\mathrm{Ca}^{2+}$ influx. Curr Biol 15, 1235-1241 (2005).

3. Feske, S. et al. A mutation in Orail causes immune deficiency by abrogating CRAC channel function. Nature 441, 179-185 (2006).

4. Vig, M. et al. CRACM1 multimers form the ion-selective pore of the CRAC channel. Curr Biol 16, 2073-2079 (2006).

5. Zhang, S. L. et al. Genome-wide RNAi screen of $\mathrm{Ca}^{2+}$ influx identifies genes that regulate $\mathrm{Ca}^{2+}$ release-activated Ca ${ }^{2+}$ channel activity. Proc Natl Acad Sci USA 103, 9357-9362 (2006).

6. Hoth, M. \& Penner, R. Calcium release-activated calcium current in rat mast cells. J Physiol 465, 359-386 (1993).

7. Derler, I. et al. A Ca ${ }^{2+}$ Release-activated $\mathrm{Ca}^{2+}$ (CRAC) Modulatory Domain (CMD) within STIM1 Mediates Fast Ca ${ }^{2+}$-dependent Inactivation of ORAI1 Channels. J Biol Chem 284, 24933-24938 (2009).

8. Zweifach, A. \& Lewis, R. S. Slow calcium-dependent inactivation of depletion-activated calcium current. Store-dependent and -independent mechanisms. J Biol Chem 270, 14445-14451 (1995).

9. Parekh, A. B. Slow feedback inhibition of calcium release-activated calcium current by calcium entry. J Biol Chem 273, 14925-14932 (1998).

10. Stathopulos, P. B., Zheng, L., Li, G. Y., Plevin, M. J. \& Ikura, M. Structural and mechanistic insights into STIM1-mediated initiation of store-operated calcium entry. Cell 135, 110-122 (2008).

11. Yuan, J. P., Zeng, W., Dorwart, M. R., Choi, Y. J., Worley, P. F. \& Muallem, S. SOAR and the polybasic STIM1 domains gate and regulate Orai channels. Nat Cell Biol 11, 337-343 (2009).

12. Muik, M. et al. A Cytosolic Homomerization and a Modulatory Domain within STIM1 C Terminus Determine Coupling to ORAI1 Channels. J Biol Chem 284, 8421-8426 (2009).

13. Park, C. Y. et al. STIM1 clusters and activates CRAC channels via direct binding of a cytosolic domain to Orail. Cell 136, 876-890 (2009).

14. Kawasaki, T., Lange, I. \& Feske, S. A minimal regulatory domain in the C terminus of STIM1 binds to and activates ORAI1 CRAC channels. Biochem Biophys Res Commun 385, 49-54 (2009).

15. Muik, M. et al. STIM1 couples to ORAI1 via an intramolecular transition into an extended conformation. EMBO J 30, 1678-1689 (2011).

16. Yang, X., Jin, H., Cai, X., Li, S. \& Shen, Y. Structural and mechanistic insights into the activation of Stromal interaction molecule 1 (STIM1). Proc Natl Acad Sci USA 109, 5657-5662 (2012).

17. Jha, A. et al. The STIM1 CTID domain determines access of SARAF to SOAR to regulate Orail channel function. J Cell Biol 202, 71-79 (2013)

18. Palty, R., Raveh, A., Kaminsky, I., Meller, R. \& Reuveny, E. SARAF inactivates the store operated calcium entry machinery to prevent excess calcium refilling. Cell 149, 425-438 (2012). 
19. Albarran, L., Lopez, J. J., Dionisio, N., Smani, T., Salido, G. M. \& Rosado, J. A. Transient receptor potential ankyrin-1 (TRPA1) modulates store-operated $\mathrm{Ca}^{2+}$ entry by regulation of STIM1-Orail association. Biochim Biophys Acta 1833, 3025-3034 (2013).

20. Csutora, P. et al. Novel role for STIM1 as a trigger for calcium influx factor production. J Biol Chem 283, 14524-14531 (2008).

21. Albarran, L. et al. TRPC6 participates in the regulation of cytosolic basal calcium concentration in murine resting platelets. Biochim Biophys Acta 1843, 789-796 (2014).

22. Lopez, J. J., Salido, G. M., Pariente, J. A. \& Rosado, J. A. Interaction of STIM1 with endogenously expressed human canonical TRP1 upon depletion of intracellular $\mathrm{Ca}^{2+}$ stores. J Biol Chem 281, 28254-28264 (2006).

23. Huang, G. N. et al. STIM1 carboxyl-terminus activates native SOC, I(crac) and TRPC1 channels. Nat Cell Biol 8, 1003-1010 (2006).

24. Shuttleworth, T. J., Thompson, J. L. \& Mignen, O. STIM1 and the noncapacitative ARC channels. Cell Calcium 42, 183-191 (2007).

25. Zhang, X. et al. Complex role of STIM1 in the activation of store-independent Orail/3 channels. J Gen Physiol 143, 345-359 (2014).

26. Yuan, J. P. et al. TRPC channels as STIM1-regulated SOCs. Channels (Austin) 3, 221-225 (2009).

27. Zeng, W. et al. STIM1 gates TRPC channels, but not Orail, by electrostatic interaction. Mol Cell 32, 439-448 (2008).

28. Cheng, K. T., Liu, X., Ong, H. L. \& Ambudkar, I. S. Functional requirement for Orail in store-operated TRPC1-STIM1 channels. J Biol Chem 283, 12935-12940 (2008).

29. Ong, H. L. et al. Dynamic assembly of TRPC1-STIM1-Orail ternary complex is involved in store-operated calcium influx. Evidence for similarities in store-operated and calcium release-activated calcium channel components. J Biol Chem 282, 9105-9116 (2007).

30. Jardin, I., Lopez, J. J., Salido, G. M. \& Rosado, J. A. Orail mediates the interaction between STIM1 and hTRPC1 and regulates the mode of activation of hTRPC1-forming $\mathrm{Ca}^{2+}$ channels. J Biol Chem 283, 25296-25304 (2008).

31. Maleth, J., Choi, S., Muallem, S. \& Ahuja, M. Translocation between PI(4,5)P2-poor and PI(4,5)P2-rich microdomains during store depletion determines STIM1 conformation and Orail gating. Nat Commun 5, 5843 (2014).

32. Pani, B. et al. Impairment of TRPC1-STIM1 channel assembly and AQP5 translocation compromise agonist-stimulated fluid secretion in mice lacking caveolin1. J Cell Sci 126, 667-675 (2013).

33. $\mathrm{Ng}, \mathrm{L}$. C. et al. TRPC1 and Orail interact with STIM1 and mediate capacitative $\mathrm{Ca}^{2+}$ entry caused by acute hypoxia in mouse pulmonary arterial smooth muscle cells. Am J Physiol Cell Physiol 303, C1156-1172 (2012).

34. Jardin, I., Salido, G. M. \& Rosado, J. A. Role of lipid rafts in the interaction between hTRPC1, Orai1 and STIM1. Channels (Austin) 2, 401-403 (2008).

35. Derler, I. et al. Cholesterol modulates Orail channel function. Sci Signal 9, ra10 (2016).

36. Lopez, E., Berna-Erro, A., Salido, G. M., Rosado, J. A. \& Redondo, P. C. FKBP52 is involved in the regulation of SOCE channels in the human platelets and MEG 01 cells. Biochim Biophys Acta 1833, 652-662 (2013).

37. Dionisio, N., Smani, T., Woodard, G. E., Castellano, A., Salido, G. M. \& Rosado, J. A. Homer proteins mediate the interaction between STIM1 and Cav1.2 channels. Biochim Biophys Acta 1853, 1145-1153 (2015).

38. Albarran, L., Lopez, J. J., Woodard, G. E., Salido, G. M. \& Rosado, J. A. Store-operated Ca ${ }^{2+}$ entry-associated regulatory factor (SARAF) plays an important role in the regulation of arachidonate-regulated $\mathrm{Ca}^{2+}$ (ARC) channels. J Biol Chem 291, 6982-6988 (2016).

39. Woodard, G. E., Lopez, J. J., Jardin, I., Salido, G. M. \& Rosado, J. A. TRPC3 regulates agonist-stimulated Ca ${ }^{2+}$ mobilization by mediating the interaction between type I inositol 1,4,5-trisphosphate receptor, RACK1, and Orai1. J Biol Chem 285, 8045-8053 (2010).

40. Redondo, P. C., Ben-Amor, N., Salido, G. M., Bartegi, A., Pariente, J. A. \& Rosado, J. A. Ca ${ }^{2+}$-independent activation of Bruton's tyrosine kinase is required for store-mediated $\mathrm{Ca}^{2+}$ entry in human platelets. Cell Signal 17, 1011-1021 (2005).

41. Gruszczynska-Biegala, J. \& Kuznicki, J. Native STIM2 and ORAI1 proteins form a calcium-sensitive and thapsigargin-insensitive complex in cortical neurons. J Neurochem 126, 727-738 (2013).

\section{Acknowledgements}

Supported by MINECO (Grants BFU2013-45564-C2-1-P, BFU2013-45564-C2-2-P, BFU2011-24365 and RD120043-0016 (RETICEF)) and Junta de Extremadura-FEDER. JJL and LA are supported by Juan de la Cierva Program and MINECO fellowship BES-2011-043356, respectively. The pEYFP-Orail mutants and pEYFP-STIM1 overexpression plasmid were kindly provided by Dr. Romanin (Johannes Kepler University, Austria).

\section{Author Contributions}

L.A., J.J.L., N.B.A. and A.B.-E. performed the experiments. FEM-C performed experiments and participated in the discussion. J.A.R. designed the study, prepared the figures and wrote the manuscript. T.S. and G.M.S. contributed to the design of the manuscript and the discussion. All authors reviewed and approved the manuscript.

\section{Additional Information \\ Supplementary information accompanies this paper at http://www.nature.com/srep}

Competing financial interests: The authors declare no competing financial interests.

How to cite this article: Albarran, L. et al. Dynamic interaction of SARAF with STIM1 and Orail to modulate store-operated calcium entry. Sci. Rep. 6, 24452; doi: 10.1038/srep24452 (2016).

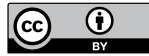

This work is licensed under a Creative Commons Attribution 4.0 International License. The images or other third party material in this article are included in the article's Creative Commons license, unless indicated otherwise in the credit line; if the material is not included under the Creative Commons license, users will need to obtain permission from the license holder to reproduce the material. To view a copy of this license, visit http://creativecommons.org/licenses/by/4.0/ 